\title{
Three-dimensional echocardiographic virtual endoscopy for the diagnosis of congenital heart disease in children
}

\author{
Haihong Xue $\cdot$ Kun Sun $\cdot$ Jianguo Yu $\cdot$ \\ Binjin Chen • Guozhen Chen · Wenjing Hong • \\ Liping Yao $\cdot$ Lanping Wu
}

Received: 12 November 2009/Accepted: 27 May 2010/Published online: 10 June 2010

(C) The Author(s) 2010. This article is published with open access at Springerlink.com

\begin{abstract}
Virtual endoscopy (VE) is a new postprocessing method that uses volumetric data sets to simulate the tracks of a "conventional" flexible endoscope. However, almost all studies of this method have involved virtual visualizations of the cardiovascular structures applied to computed tomography (CT) and magnetic resonance (MR) datasets. This paper introduces a novel visualization method called the "three-dimensional echocardiographic intracardiac endoscopic simulation system (3DE IESS)", which uses 3D echocardiographic images in a virtual reality (VR) environment to diagnose congenital heart disease. The aim of this study was to analyze the feasibility of VE in the evaluation of congenital heart
\end{abstract}

H. Xue $\cdot$ K. Sun $(\bowtie)$

Department of Pediatric Cardiology, Xinhua Hospital

Affiliated to Shanghai Jiaotong University School of

Medicine, Shanghai 200092, China

e-mail: drkunsun@yahoo.com.cn

K. Sun · G. Chen · W. Hong · L. Wu

Department of Pediatric Cardiology, Shanghai Children's Medical Center Affiliated to Shanghai Jiaotong University School of Medicine, Shanghai 200127, China

J. Yu $\cdot$ B. Chen

Department of Electronic Engineering, Fudan University, Shanghai 200433, China

\section{Yao}

Department of Ultrasound, Xinhua Hospital Affiliated to Shanghai Jiaotong University School of Medicine,

Shanghai 200092, China disease in children and its accuracy compared with 2DE. Three experienced pediatric cardiologists blinded to the patients' diagnoses separately reviewed 40 two-dimensional echocardiographic (2DE) datasets and 40 corresponding VE datasets and judged whether abnormal intracardiac anatomy was present in terms of a five-point scale $(1=$ definitely absent; 2 = probably absent; $3=$ cannot be determined; $4=$ probably present; and $5=$ definitely present). Compared with clinical diagnosis, the diagnostic accuracy of VE was 98.7\% for ASD, $92.4 \%$ for VSD, $92.6 \%$ for TOF, and $94 \%$ for DORV, respectively. Diagnostic accuracy of VE was significantly higher than that of $2 \mathrm{DE}$ for TOF and DORV except for ASD and VSD. The receiver operating characteristic (ROC) curve for VE was closer to the optimal performance point than was the ROC curve for 2DE. The area under the ROC curve was 0.96 for $\mathrm{VE}$ and 0.93 for 2DE. Kappa values (range, 0.73-0.79) for VE and 2DE indicated substantial agreement. 3D echocardiographic VE can enhance our understanding of intracardiac structures and facilitate the evaluation of congenital heart disease.

Keywords Three-dimensional echocardiography . Virtual endoscopy · Virtual reality .

Congenital heart disease

\section{Introduction}

Congenital heart disease is the most common cause of death in infants, stimulating increased interest in 
developing appropriate diagnostic tools for the reliable detection of congenital heart malformations. Two-dimensional echocardiography (2DE) has been the main noninvasive technique used in the diagnosis of congenital heart disease. However, given that the heart has a complex anatomy and is in constant motion, conventional 2DE can provide only partial information about the spatial and temporal relationships between cardiac structures. In addition, most cardiologists are familiar with 2D imaging and mentally reconstruct stereoscopic images of the heart, relying primarily on experience and medical knowledge. Three-dimensional echocardiography (3DE) may provide valuable information that is not obtainable with conventional 2DE and that can enhance our understanding of the complex anatomy of cardiac structures [1-3].

Virtual endoscopy (VE) can simulate the tracks of a "conventional" endoscope using 3D image datasets, such as those from computed tomography (CT), magnetic resonance (MR), or echo scans [4]. Preliminary studies have suggested that VE can provide accurate and reproducible visualizations [5]. Virtual visualizations of the cardiovascular structures have been considered to be more realistic and useful, compared with standard endoscopic views [6]. However, almost all studies have used volumetric CT and MR data [7-11]; few have dealt with echocardiography data [12-14]. The rapidly increasing power of computer hardware and software has led to the use of virtual reality (VR) as a technique for medical visualization in the past decade. Technologically advanced 3D reconstruction software has multiple advantages over traditional imaging modalities for the evaluation of congenital heart malformations. With the ongoing revolution in the computer industry, static and dynamic VE visualization of volumetric three-dimensional echocardiography datasets is becoming possible.

This paper introduces a novel approach for the visualization of dynamic 3DE data. In close cooperation with the electronic engineering department of Fudan University, we have developed a VE system called the "three-dimensional echocardiographic intracardiac endoscopic simulation system" (3DE IESS). We have previously applied VR techniques for clinical research purposes [13]. Our approach is based on a tool for the interactive VR visualization of $3 \mathrm{DE}$ datasets in the form of $3 \mathrm{D}$ heart models displayed on VR equipment. VR can assist with the interpretation of $3 \mathrm{D}$ data for heart volume and facilitates the development of 3D heart models [15]. In this pilot study, we have evaluated the ability of 3DE IESS to reconstruct the inner-cardiac structures. The purpose of this study was to assess whether VE is feasible for 3DE and to determine if 3DE imaging in a virtual environment can be used effectively as a clinical diagnostic tool.

\section{Methods}

Study population

This protocol was approved by Xinhua Hospital Affiliated to Shanghai Jiaotong University School of Medicine (Shanghai 200092, China), and have therefore been performed in accordance with the ethical standards laid down in the 1964 Declaration of Helsinki and its later amendments.

Datasets from 13 healthy subjects and 35 patients with congenital heart disease were collected. There were 30 males and 18 females, with a median age of 3.8 years (range: $0.2-8.5$ years).

Data acquisition and processing

After conventional transthoracic echocardiography, data acquisition for 3DE was performed. The 3DE image datasets were generated using a 2- to $4-\mathrm{MHz}$ matrix array transducer in a Philips Sonos 7500 echocardiographic system (Philips Medical Systems, Andover, MA, USA) equipped with a 3D dataacquisition software package. After acquisition, the echocardiographic 3D images were stored on CD-ROM and transferred to a computer for postprocessing and data analysis.

The images were evaluated with respect to subjective image quality based on possible anatomical differentiation of heart tissues. All available images were graded as below: insufficient (poor quality with step artifacts and stripes throughout the image limiting evaluation of the heart), sufficient (sufficient quality with step artifacts and stripes which have minor implication on the evaluation of the heart), good (good quality with minor motion artifacts not hampering the evaluation of the heart) or optimal (excellent quality without motion artifacts). 
VE reconstructions

The cardiac structures were reconstructed after preprocessing. The intracardiac anatomy was assessed using 3DE IESS, which provides a flexible framework for intuitive visualization and interactive evaluation of large medical datasets. The interior of the heart was examined using VE. To secure relevant examinations of all details related to intracardiac morphology, the heart model can be reproducibly visualized, and the virtual eye can be installed at any point inside the heart.

The software for our echocardiographic analysis system was written in Visual $\mathrm{C}++6.0$ and employed graphics and visualization utilities available in the VTK (Visualization ToolKit, Kitware, Inc.). Color and lighting were used to enhance realism.

Visualization in a virtual reality environment

In the VR environment, the observer is within the heart, close to anatomical structures in regions of interest, and can move slowly around objects of interest. Slightly angling or rotating the virtual eye in various projections can produce specific and unique imaging. By controlling the view with the mouse, the operator can move the object of interest. Primarily head-on views of the intracardiac anatomy from the atrium and ventricle were reconstructed to determine the precise anatomy of the heart.

Three experienced pediatric cardiologists blinded to the patients' diagnoses separately reviewed the 40 2DE datasets and 40 corresponding VE datasets. Data sheets were used to record the results of their findings. Clinical diagnoses of healthy hearts were conducted using 2D Doppler transthoracic echocardiography. Surgical findings served as the gold standard for the clinical diagnoses of congenital heart malformations. The findings were relevant to a group of patients who had undergone surgery for congenital heart disease, rather than patients who had not had a surgical procedure. The cardiologists judged whether abnormal intracardiac anatomy was present on a fivepoint scale $(1=$ definitely absent; $2=$ probably absent; $3=$ cannot be determined; $4=$ probably present; and $5=$ definitely present). Three observers independently examined each of the $2 \mathrm{D}$ and $\mathrm{VE}$ datasets to provide 162 diagnostic evaluations of each method.
Statistical analysis

The results of the area under the receiver operating characteristic (ROC) curve for VE echocardiography and 2DE were analyzed using SPSS 13.0 for Windows (SPSS, Inc. Chicago, IL). Agreement for interobserver variability was analyzed by computing the kappa values.

\section{Results}

Of the 48 patients enrolled, the three-dimensional reconstruction analyzed by 3 DE IESS was sufficient in 40 patients with good or optimal image quality, thus the feasibility was $83.3 \%$. It was not feasible in 8 patients $(16.7 \%)$ due to poor image quality failure in reconstruction. Reconstruction analyzed by $3 \mathrm{DE}$ IESS was sufficient for all 40 patients. An overview of the selected patients is shown in Table 1 .

The mean time for VE reconstruction was approximately $5 \mathrm{~min}$. The time needed for laborious manual data analysis ranged from 30 to $60 \mathrm{~min}$.

Images of the atrial septum were captured from either the right or left atrial sides. VE was oriented towards the atrial septal surface, and a left atrial headon view could be observed. Similarly, the interventricular septum could be visualized head-on from either the left or right ventricles (Figs. 1, 2, 3, 4).

VE displayed dynamic, continuous images for evaluation with a realistic perspective, giving the impression of a 3D image (Fig. 5). In the dynamic

Table 1 Summary of study patient diagnoses

\begin{tabular}{lc}
\hline Diagnoses & $\begin{array}{l}\text { No. of } \\
\text { patients }\end{array}$ \\
\hline Normal & 10 \\
ASD & 9 \\
VSD & 4 \\
TOF & 4 \\
ASD/VSD & 2 \\
TOF/ASD & 4 \\
DORV/VSD & 5 \\
DORV/AVSD & 1 \\
DORV/VSD/ASD & 1 \\
\hline
\end{tabular}

$A S D$ atrial septal defect, $V S D$ ventricular septal defect, TOF tetralogy of Fallot, DORV double outlet right ventricle, $A V S D$ atrioventricular septal defect 


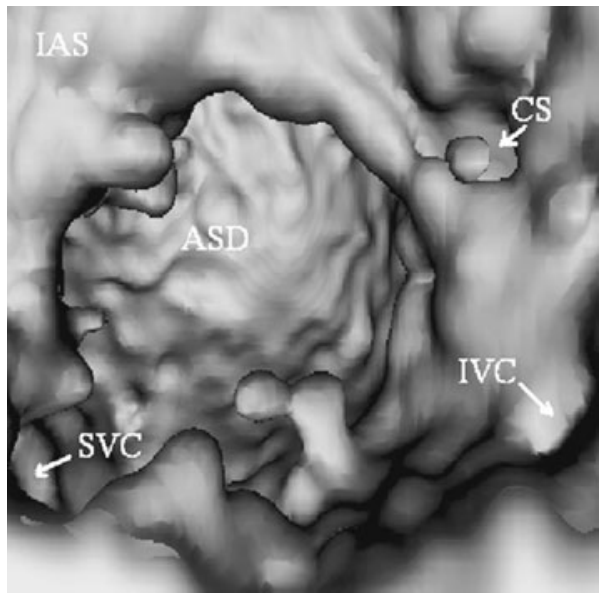

Fig. 1 Virtual endoscopic view of ASD from the right atrium by 3DE IESS to image the relationships among the ASD, IVC, $\mathrm{SVC}$, and CS. $A S D$ atrial septal defect, $I V C$ inferior vena cava, $S V C$ superior vena cava, $C S$ coronary sinus

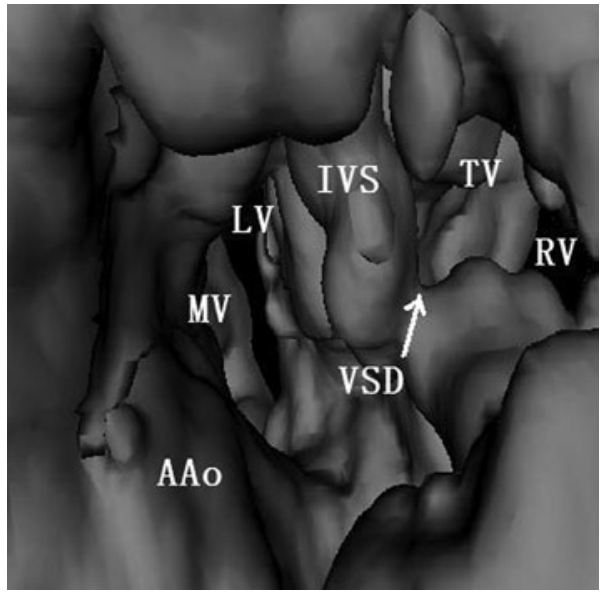

Fig. 2 Virtual endoscopic view of tetralogy of Fallot from the ascending aorta toward the interventricular septum by $3 \mathrm{DE}$ IESS. $L V$ left ventricle, $R V$, right ventricle; $A A o$ ascending aorta, IVS interventricular septum, $V S D$ ventricular septal defect; $M V$ mitral valve, $T V$ tricuspid valve

mode display, the opening and closing of the cardiac valves can be easily visualized. Moreover, valve motion in any desired phase of the cardiac cycle can also be observed.

Compared with clinical diagnosis, the accuracy of VE was $98.7 \%$ for ASD, $92.4 \%$ for VSD, $92.6 \%$ for TOF, and 94\% for DORV, respectively. Diagnostic accuracy of VE was significantly higher than that of 2DE for TOF and DORV except for ASD and VSD (Table 2).

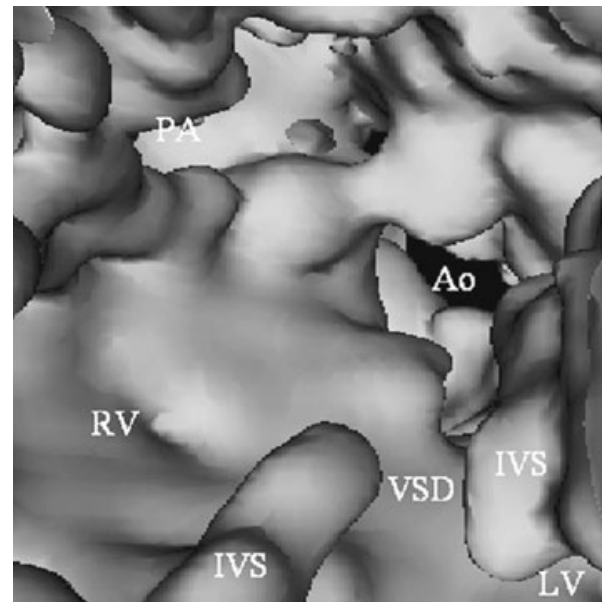

Fig. 3 Virtual endoscopic view of double outlet right ventricle viewed above the interventricular septum by 3DE IESS. $L V$ left ventricle, $R V$ right ventricle, IVS interventricular septum, VSD ventricular septal defect; $A o$ aorta, $P A$ pulmonary artery

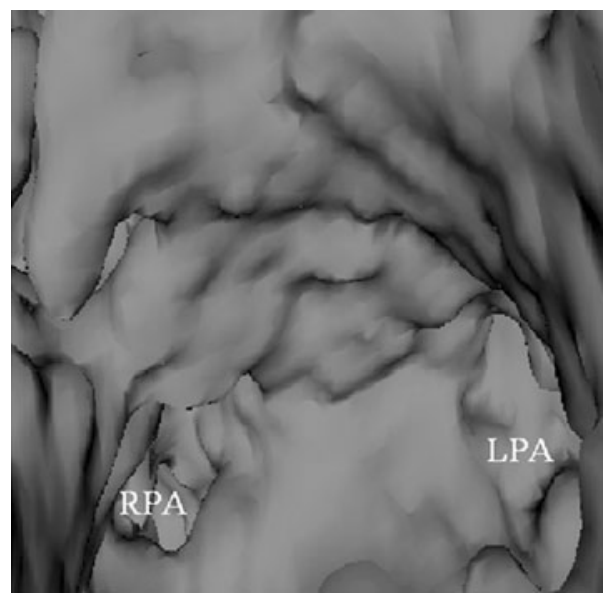

Fig. 4 Virtual endoscopic view of a normal heart from the pulmonary artery by 3DE IESS, used to image the relationship between LPA and RPA. LPA left pulmonary artery, RPA right pulmonary artery

The results of clinical evaluations of the 132 innercardiac abnormalities and 30 normal cases by three observers for the 2DE images are shown in Table 3. Among the 132 congenital heart abnormalities, only $89(67.4 \%)$ studies were identified as "definitely present", $12(9.1 \%)$ as "probably present", $11(8.3 \%)$ as "cannot determine" and $4(3.0 \%)$ as "probably absent". However, 16 (12.1\%) images were mistakenly identified as "definitely absent" of abnormalities. Twenty-six $(86.7 \%)$ of the 30 normal cases were 

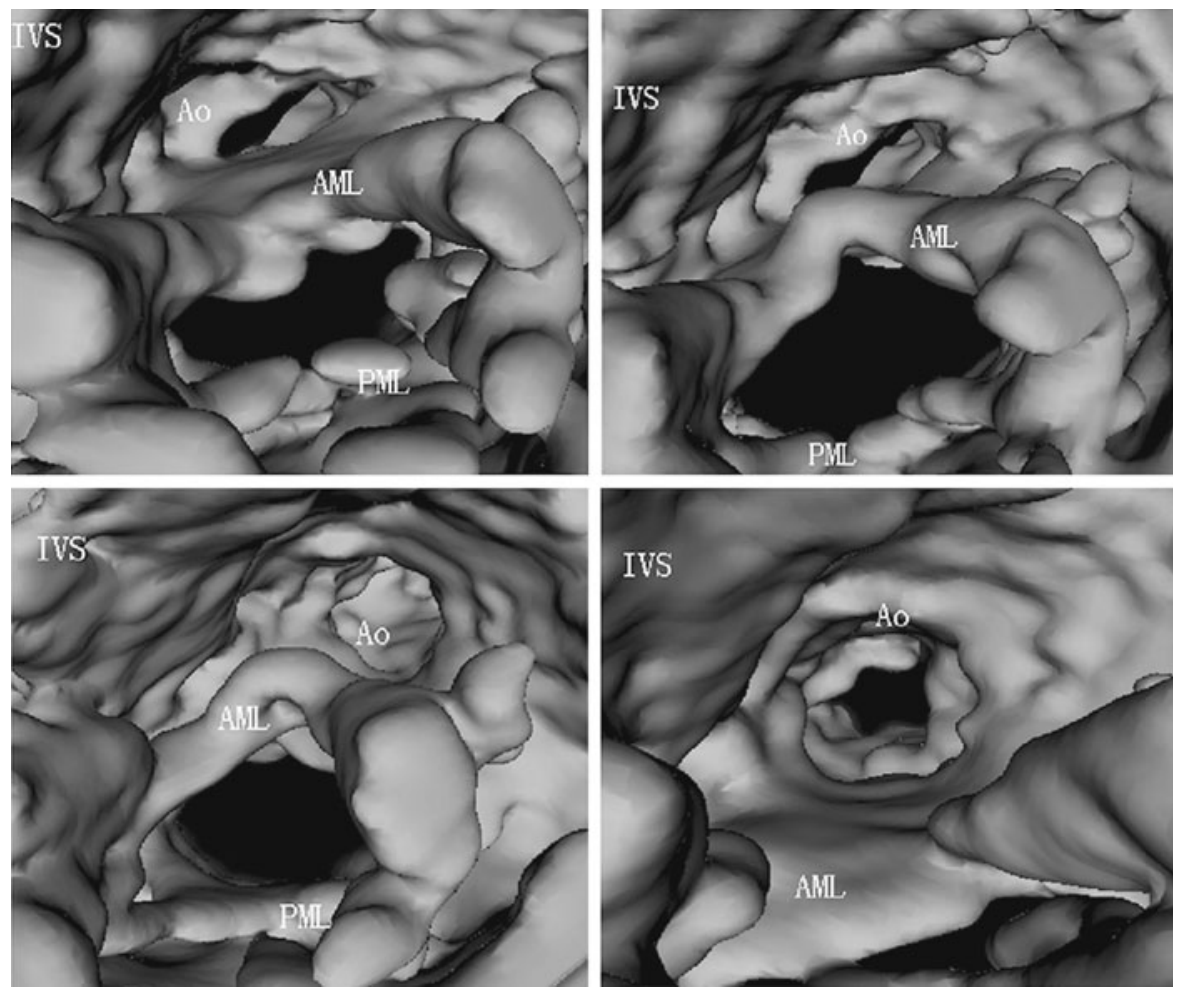

Fig. 5 Multiphase reconstructed images of intracardiac structures (viewed from the left ventricle) in the cardiac cycle. $A M L$ anterior mitral leaflet, $P M L$ posterior mitral leaflet, Ao aorta, IVS interventricular septum

Table 2 Incremental diagnostic value of VE over 2DE

\begin{tabular}{llll}
\hline Diagnoses & \multicolumn{2}{l}{ Accuracy } & $P$ value \\
\cline { 2 - 3 } & VE $(\%)$ & 2DE $(\%)$ & \\
\hline ASD & 98.7 & 97.4 & 0.495 \\
VSD & 92.4 & 95.5 & 0.357 \\
TOF & 92.6 & 88.2 & 0.048 \\
DORV & 94.0 & 86.2 & 0.032 \\
\hline
\end{tabular}

Table 3 Results of clinical evaluations for data presented as 2D echocardiographic images

\begin{tabular}{lcc}
\hline Category & Group & \\
\cline { 2 - 3 } & Abnormal & Normal \\
\hline 1 Definitely absent & 16 & 26 \\
2 Probably absent & 4 & 1 \\
3 Cannot determine & 11 & 2 \\
4 Probably present & 12 & 1 \\
5 Definitely present & 89 & 0 \\
Total & 132 & 30 \\
\hline
\end{tabular}

identified as "definitely absent" of congenital heart defects. Other ratings demonstrated uncertainty or confusion about the true state of the cardiac structures to various degree. Table 4 illustrates the results of the same observers for the 3D VE images. Better performance was achieved. VE demonstrated clinically significant abnormalities in $92(69.7 \%)$ of the 132 inner-cardiac abnormalities. Only $3(2.3 \%)$ of the 132 abnormal and $1(3.3 \%)$ of the 30 normal cases

Table 4 Results of clinical evaluations for data presented as 3D echocardiographic virtual endoscopy images

\begin{tabular}{lcc}
\hline Category & Group & \\
\cline { 2 - 3 } & Abnormal & Normal \\
\hline 1 Definitely absent & 3 & 22 \\
2 Probably absent & 3 & 4 \\
3 Cannot determine & 14 & 1 \\
4 Probably present & 20 & 2 \\
5 Definitely present & 92 & 1 \\
Total & 132 & 30 \\
\hline
\end{tabular}




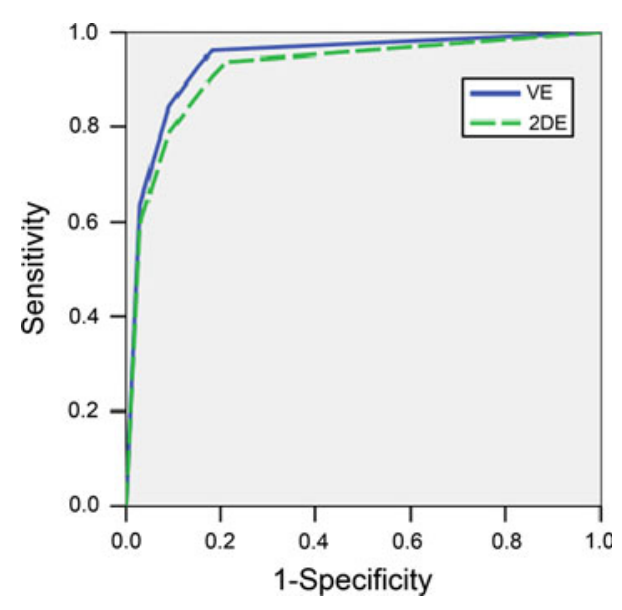

Fig. 6 Receiver operating characteristic (ROC) curves for VE (solid) and 2DE (dashed)

Table 5 Observer agreement for diagnosis based on echocardiography

\begin{tabular}{lll}
\hline Observer combinations & 2DE & VE \\
\hline Observer 1 and 2 & 0.76 & 0.73 \\
Observer 1 and 3 & 0.79 & 0.79 \\
Observer 2 and 3 & 0.73 & 0.76 \\
\hline
\end{tabular}

were mistakenly identified as "definitely absent" or "definitely present", respectively.

ROC curves for 2DE and VE were constructed, and the area under the curve for each echocardiographic image was compared. As shown in Fig. 6, the ROC curve for VE (solid line) was closer to the optimal performance point (upper left corner) than the ROC curve for 2DE (dashed line). The area under the ROC curve was 0.96 for VE and 0.93 for 2DE.

\section{Inter-observer variability}

Strong inter-observer agreement was observed in this study. Kappa values (range: 0.73-0.79) for VE and 2DE indicated substantial agreement (Table 5).

\section{Discussion}

Although traditional 2DE may provide important information about the spatial and temporal relationships of cardiac anatomy during the cardiac cycle, specific details may be lacking. The complexity of cardiac anatomy requires the 3D spatial orientation of images to achieve a better understanding of the heart structures. In addition, conventional echocardiography requires a mental conceptualization process by the cardiologist to reconstruct a complex 3D anatomy image of the heart based on an interpretation of multiple 2D slices.

3D echocardiography may provide more reliable information than $2 \mathrm{D}$ methods and may improve the comprehension of anatomical relationships, especially for complex congenital heart diseases [16]. Real-time 3DE (RT-3DE) has proven to be valuable for better evaluation of morphologic abnormalities and comprehension of the complex cardiac anatomy involved in congenital heart disease [17-19]. However, RT-3DE volume-rendered images often pose difficulties in understanding both the origin and orientation of reconstructed tomographic views. Viewing heart images is analogous to outdoor observation: one cannot observe the inside structures without removing, at least partially, the walls. In order to display the intracardiac anatomy, the heart must be cut open using a cutting plane, and the images beyond this plane must be reconstructed as if the heart were cut open via surgery.

While the 3DE reconstructions can only be presented on a flat 2D screen, VR allows for visualization of the actual 3D anatomy of the heart. Lee [20] proposed the idea of applying virtual ultrasound simulations in 3DE in 1996 and reported a study on a unique computer-aided interactive 3D heart model with virtual ultrasound simulation for fetal ultrasound training. The Fetal Imaging Workstation Demonstration created the illusion of a scanning plane coursing through a 3D ultrasound heart model via pre-rendered frame animations, special effects, and solid constructive geometry. Physicians may be able to visualize, scan, navigate around, manipulate, and measure the volume of a reconstructed $3 \mathrm{D}$ virtual heart through virtual ultrasound simulation. VR can effectively reconstruct a virtual heart model and provide spatial cardiac information [15]. Some congenital heart diseases, such as ventricular septal defects and atrial septal defects (VSDs and ASDs, respectively), have been diagnosed, analyzed, or assessed within a virtual reality heart using the software module TiDAS (Toolkit for the Interactive Design of Animation and Simulation) [12]. In 2005, Bosch et al. [14] 
described dynamic 3D echocardiography using virtual reality and showed that dynamic holographic imaging of 3DE data was feasible.

The results show that reconstruction from VE can be achieved with high accuracy and low artificial variability in patients with good even optimal echocardiographic image quality. In this study, we demonstrated that 3DE can serve as a clinical tool for VE, whereas most papers published to date have exclusively used CT and MR. For these purposes, we recently introduced a novel method called the "threedimensional echocardiographic intracardiac endoscopic simulation system" or 3DE IESS. Employing virtual reality to capture realistic views of the cardiac anatomy, the 3DE IESS is an innovative approach for noninvasive imaging of the intracardiac structures. VE allows cardiologists and cardiac surgeons to examine the model as if they were actually entering into the $3 \mathrm{D}$ anatomy of the heart themselves. In the stereoscopic reconstructed image, the interior of the heart can be clearly visualized at different levels, similar to a real intracardiac endoscopic image. To secure relevant examination of all details related to cardiac morphology, the viewpoint can be rescaled and the "virtual eye" can be repositioned to any point inside the heart. In addition, the 3DE IESS can usually be installed on a computer.

VE can produce unconventional views of congenital heart disease and improve our understanding of intracardiac malformations, as an orthogonal view may be difficult to display using conventional echocardiography. The interatrial and interventricular septum can be visualized head-on, providing for better intuition concerning the relevant spatial relationships with neighboring structures. In a view from the inside of the right atrium, the spatial relationship of a defect to the superior vena cava becomes readily evident when the virtual eye is in a superior and posterior orientation. Visualization of a more unusual inferior and posterior secundum atrial septum is obtained by manipulation of the virtual eye and rotation of the 3D image inferior and posterior to the inferior vena cava. Likewise, the entrance of the coronary sinus into the atrium and its correlation to atrial structures can be similarly visualized.

Many clinical and experimental studies have demonstrated that VR is now ready for clinical application. Employing virtual reality, 3DE allows for a better understanding of the position of cardiac structures in relation to one another [12-15, 21, 22]. The unique ability of VE to depict the intracardiac anatomy from both the atrium and the ventricle, with access to head-on views, allows for better anatomical definition in relation to adjacent cardiac structures. This novel method can provide clinicians with more confidence in diagnoses of the complex anatomy of congenital heart disease. Additionally, VE can provide accurate and reproducible visualizations. Its versatility allows observers to retrieve an infinite number of different views of the intracardiac anatomy following the examination procedure.

VE offers great potential for aiding in complex diagnostic situations, assisting in planning surgical procedures, and in postoperative assessments [23]. VE is particularly helpful because it enables the heart to be visualized from the same perspective that the surgeon would see intraoperatively. Virtual reality visualization may lead to a better understanding of the intracardiac anatomy and facilitate the accuracy of preoperative planning.

Virtual reality also provides a unique resource for cardiac disease education and can add to the existing knowledge of complex pathology. Weidenbach et al. $[24,25]$ introduced a simulator (EchoComJ) with an intelligent training system to support trainees in adjusting to standard echocardiographic views. It may be helpful to convey or demonstrate intracardiac malformations to those unfamiliar with $2 \mathrm{DE}$ viewsespecially cardiologists, for whom detailed knowledge of the intracardiac anatomy is essential.

Although VE provides a solid overview of the completely acquired volume, not all structures of interest can be easily observed, as 3D imaging requires new knowledge of image interpretation, and thus there is a learning curve involved, particularly for less experienced operators, that should be accounted for before the full advantage of this new modality can be realized. One problem with VE is that operators must be experienced in anatomy, radiology, computer science, and clinical evaluation.

\section{Study limitations}

There are some limitations to this study. One major limitation of VE with the existing technology is the time-consuming nature of the off-line processing. VE image obtained with the commercially available equipment provided images with sufficient detail 
transferred to a separate workstation for off-line data analysis. The mean time for VE reconstruction was approximately $5 \mathrm{~min}$. Even though, the interpretation of 3D images are time consuming and dependent on the complexity of the intracardiac structures. Laborious manual data analysis in a number of selected ideal endoscopic views, however, is time-consuming. In the majority of patients, off-line image processing and tracing required approximately 30-60 min.

Another limitation is the possible difficulty in navigating. In several instances during this study, observers lost their sense of orientation while 'inside' the intracardiac anatomy. Understanding the orientation of a 3D visualization of echocardiographic images is often difficult due to the lack of visible anatomical landmarks. A navigation system would be sufficient in resolving the disorientation problems [26].

There are still some limitations on visualization due to artifacts of moving heart and moving floppy heart structures. 3D reconstruction of VE depends on the quality of the original images of which judged on the presence or absence of artifacts throughout the cardiac cycle. The acquisition of multiple cardiac cycles may cause stitch artifacts. Poor acoustic window and minor patients' movement can also result in artifacts. Additionally, only the 2D TTE images were selected for analysis. It is possible that the 2D transthoracic echocardiogram might not capture the clinical diagnosis of a congenital heart disease, whereas a transesophageal matrix array transducer probably does not incur problems with acoustic shadowing and can provide images of good quality. Even though this is a potential limitation of the study, it does not have much of an effect from our perspective because only high quality transthoracic 2DE and VE images were included in the study. Our initial research demonstrated that 3DE can serve as a clinical tool for VE. Further studies are necessary to improve image quality and "reality" of endoscopic mode images.

Moreover, the study is limited by the lack of a gold standard for the diagnosis. Although clinical findings served as the gold standard for the clinical diagnoses of congenital heart defect, clinical diagnosis of normal hearts was made using 2D Doppler transthoracic echocardiography.

Admittedly, the study is limited by the relatively small sample size, as only 40 patients were compared between the $2 \mathrm{D}$ and $3 \mathrm{D}$ image analyses. However, the conclusion of the study should not be weakened by the small number of patients. Moreover, they were not analyzed in a quantitative pattern, which prevents robust assessment.

\section{Conclusions}

We have demonstrated that three-dimensional echocardiographic virtual endoscopy can enhance our understanding of intracardiac structures and provide intuitive, reproducible, and clinically useful visualizations. Virtual reality techniques may provide important information by specifying the spatial orientation of the intracardiac anatomy, versus standard 2DE. This technique can aid cardiologists by adding many two-dimensional slices to accurately form three-dimensional objects from these data. The role of 3DE VE in clinical practice has yet to be clarified. However, this preliminary VE study allows us to speculate on situations in which this novel method may be useful.

Virtual reality expands the capabilities of noninvasive cardiology and may open new doors for the evaluation of congenital heart diseases. In order to accomplish this, prospective studies will be needed.

Acknowledgments This work was supported by the National Natural Scientific Foundation of China (No. 30371497). The authors thank Dean Ta (Fudan University) for reviewing the article.

Conflict of interest statement The authors declare that they have no conflict of interests.

Open Access This article is distributed under the terms of the Creative Commons Attribution Noncommercial License which permits any noncommercial use, distribution, and reproduction in any medium, provided the original author(s) and source are credited.

\section{References}

1. Szili-Torok T, Jordaens LJ, Bruining N, Ligthart J, Roelandt JR (2003) Dynamic three-dimensional echocardiography offers advantages for specific site pacing. Circulation 107:e30

2. Pepi M, Tamborini G, Maltagliati A, Galli CA, Sisillo E, Salvi L, Naliato M, Porqueddu M, Parolari A, Zanobini M, Alamanni F (2006) Head-to-head comparison of two- and three-dimensional transthoracic and transesophageal 
echocardiography in the localization of mitral valve prolapse. J Am Coll Cardiol 48:2524-2530

3. Takahashi K, Guerra V, Roman KS, Nii M, Redington A, Smallhorn JF (2006) Three-dimensional echocardiography improves the understanding of the mechanisms and site of left atrioventricular valve regurgitation in atrioventricular septal defect. J Am Soc Echocardiogr 19:1502-1510

4. Robb RA (2000) Virtual endoscopy: development and evaluation using the visible human datasets. Comput Med Imaging Graph 24:133-151

5. Oto A (2002) Virtual endoscopy. Eur J Radiol 42:231-239

6. Szpala S, Wierzbicki M, Guiraudon G, Peters TM (2005) Real-time fusion of endoscopic views with dynamic 3-D cardiac images: a phantom study. IEEE Trans Med Imaging 24:1207-1215

7. Sorensen TS, Therkildsen SV, Makowski P, Knudsen JL, Pedersen EM (2001) A new virtual reality approach for planning of cardiac interventions. Artif Intell Med 22:193-214

8. Horiguchi J, Nakanishi T, Tamura A, Ito K, Sasaki K, Shen Y (2002) Technical innovation of cardiac multirow detector CT using multisector reconstruction. Comput Med Imaging Graph 26:217-226

9. Hsu JH, Wu DK, Chen YF, Dai ZK, Lee MH, Wu JR (2007) Virtual intraluminal evaluation of aortico-left ventricular tunnel by multislice computed tomography. Int $\mathbf{J}$ Cardiol 116:266-268

10. Wierzbicki M, Drangova M, Guiraudon G, Peters T (2004) Validation of dynamic heart models obtained using nonlinear registration for virtual reality training, planning, and guidance of minimally invasive cardiac surgeries. Med Image Anal 8:387-401

11. Kim DY, Park JW (2005) Visualization of the internal carotid artery using MRA images. Magn Reson Imaging 23:27-33

12. Bruining N, Roelandt JR, Grunst G, Berlage T, Waldinger J, Mumm B (1999) Three-dimensional echocardiography: the gateway to virtual reality!. Echocardiography 16 : 417-423

13. Sun K, Xue HH, Yu JG, Wang J, Chen GZ, Hong WJ, Wang WQ (2005) A primary exploration of three-dimensional echocardiographic intra-cardiac virtual reality visualization of atrial septal defect: in vitro validation. Comput Cardiol 32:145-146

14. van den Bosch AE, Koning AH, Meijboom FJ, McGhie JS, Simoons ML, van der Spek PJ, Bogers AJ (2005) Dynamic
3D echocardiography in virtual reality. Cardiovasc Ultrasound $3: 37$

15. Bruining N, Lancee C, Roelandt JR, Bom N (2000) Threedimensional echocardiography paves the way toward virtual reality. Ultrasound Med Biol 26:1065-1074

16. Marx GR, Sherwood MC (2002) Three-dimensional echocardiography in congenital heart disease: a continuum of unfulfilled promises? No. A presently clinically applicable technology with an important future? Yes. Pediatr Cardiol 23:266-285

17. Monaghan MJ (2006) Role of real time 3D echocardiography in evaluating the left ventricle. Heart 92:131-136

18. Lu X, Xie M, Tomberlin D, Klas B, Nadvoretskiy V, Ayres N, Towbin J, Ge S (2008) How accurately, reproducibly, and efficiently can we measure left ventricular indices using M-mode, 2-dimensional, and 3-dimensional echocardiography in children? Am Heart J 155:946-953

19. Hlavacek AM, Crawford FA Jr, Chessa KS, Shirali GS (2006) Real-time three-dimensional echocardiography is useful in the evaluation of patients with atrioventricular septal defects. Echocardiography 23:225-231

20. Lee W (1996) The fetal imaging workstation demonstration project. Comput Med Imaging Graph 20:459-466

21. Silberbach M, Sahn DJ (1993) Three-dimensional echocardiographic reconstruction: from "ice-pick" view to virtual reality. Mayo Clin Proc 68:311-312

22. Agati L (1996) Three-dimensional echocardiography: the virtual reality in cardiology-luxury or useful technique? Eur Heart J 17:487-489

23. Gallagher AG, Cates CU (2004) Virtual reality training for the operating room and cardiac catheterisation laboratory. Lancet 364:1538-1540

24. Weidenbach M, Trochim S, Kreutter S, Richter C, Berlage T, Grunst G (2004) Intelligent training system integrated in an echocardiography simulator. Comput Biol Med 34: $407-425$

25. Weidenbach M, Wild F, Scheer K, Muth G, Kreutter S, Grunst G, Berlage T, Schneider P (2005) Computer-based training in two-dimensional echocardiography using an echocardiography simulator. J Am Soc Echocardiogr 18: 362-366

26. Xie X, Tao D, Chen S, Gao S, Bi Y (2006) 3D navigation of CTVE and correction of MinIP methods in non-invasive diagnostic detection. Comput Med Imag Grap 30:383-389 